\title{
Germanica
}

\section{Les jeunes revues littéraires, avant-garde du modernisme en Suède}

Die jungen avantgardistischen Literaturzeitschriften in Schweden

\section{Georges Ueberschlag}

\section{OpenEdition}

1 Journals

Édition électronique

URL : http://journals.openedition.org/germanica/428

DOI : 10.4000/germanica.428

ISSN : 2107-0784

Éditeur

Université de Lille

\section{Édition imprimée}

Date de publication : 1 juin 1993

Pagination : $35-46$

ISSN : 0984-2632

\section{Référence électronique}

Georges Ueberschlag, "Les jeunes revues littéraires, avant-garde du modernisme en Suède »,

Germanica [En ligne], 12 | 1993, mis en ligne le 11 avril 2013, consulté le 06 octobre 2020. URL : http:// journals.openedition.org/germanica/428; DOI : https://doi.org/10.4000/germanica.428

Ce document a été généré automatiquement le 6 octobre 2020.

(c) Tous droits réservés 


\title{
Les jeunes revues littéraires, avant- garde du modernisme en Suède
}

Die jungen avantgardistischen Literaturzeitschriften in Schweden

\author{
Georges Ueberschlag
}

1 Dans le numéro un de la revue Utsikt qu'il venait de lancer en 1948, l'écrivain suédois Axel Liffner avait usé d'une formule très percutante pour définir son entreprise: " Forum för debut och debatt », « Un forum pour debuts et débats ».

2 La jeune revue d'avant-garde littéraire correspond toujours, en effet, à un rêve d'aventure intellectuelle et de libération, de refus du passé et d'espoir en des lendemains qui chantent. Son but s'appelle utopie. Même si on sait d'expérience qu'on ne l'atteindra jamais, l'aventure aura été belle.

3 Pour mener les assauts contre la forteresse de la culture établie, la jeune revue constitue l'arme par excellence. Les jeunes écrivains qui cherchent à se l'approprier, la manient cependant avec une telle fougue pour proclamer leurs audaces que souvent elle leur échappe des mains à peine brandie. Mais qu'à cela ne tienne! L'essentiel c'est de dire, non de tenir. Les liens avec la pensée anarchiste sont évidents, toujours, ce sont des alliés naturels en quelque sorte. La nouvelle littérature est révolutionnaire par essence, elle cherche à faire époque, à défricher de nouvelles terres. Toute revue d'avant-garde, en Scandinavie surtout, a les yeux fixés au-delà des frontières et cherche son inspiration dans un contexte international. Si la lutte est belle, les obstacles sont nombreux, il faut de l'argent, il faut des lecteurs, il faut des amis, toutes choses rares.

Dans les pays nordiques il n'y a pas de terme précis, ou consacré, pour désigner la jeune revue d'avant-garde littéraire. On parle parfois de "avantgardetidskrifter» ou de " unglitterär tidskrift », c'est-à-dire une revue pour un mouvement littéraire naissant. Ce dernier terme s'est surtout imposé parmi les critiques et dans les revues ellesmêmes à partir de 1950. Mais le mot semble avoir été employé pour la première fois par Artur Lundkvist en $1928^{1}$. Le terme se rattache évidemment à des précurseurs célèbres. Songeons à la "Jeune Allemagne », qui a eu son pendant Scandinave, la " Jeune Suède », regroupant les courants libéraux des années 1840 et poursuivant sa carrière jusqu’à la 
fin du XIX ${ }^{e}$ siècle. La jeunesse devenait une valeur nouvelle qu'on exaltait, également dans le domaine de l'art. Jeune devenait synonyme d'avant-gardisme, dans tous les domaines de l'art et de la civilisation.

5 La jeune revue naît souvent de la conscience de besoins stylistiques nouveaux. Besoins tellement pressants qu'on y annonce la couleur dès le premier numéro. "La rêverie éveillée comme conception de la vie ", écrivait par exemple Karin Boye dans le numéro un de Spektrum (1931). Il s'agit de lancer un message au monde, de lutter. La jeune revue épouse étroitement les hauts et les bas de la lutte, sa vie est fonction de son message. Si celui-ci s'épuise, elle meure, beaucoup plus sûrement encore que lorsque les conditions économiques sont mauvaises. L'existence d'une revue d'avant-garde est toujours dangereuse. C'est d'ailleurs pour cela qu'elle est toujours intéressante, a priori. Peu de revues savent se renouveler suffisamment pour durer. Dans quelques numéros on a épuisé les exigences, les témoignages du renouveau souhaité, l'appel à renverser les "grandes revues", ces institutions fermées où œuvrent les représentants d'une autre génération. Parmi les jeunes revues il y en a pourtant qui profitent d'une certaine durée, qui finissent même par entrer dans la cohorte des grandes, celles qui assurent à la littérature sa permanence.

Les deux lui sont nécessaires, et leur coexistence, même si elle est conflictuelle, est source d'épanouissement, de dynamisme pour une littérature nationale. La Suède des années 1940 a connu à la fois la très explosive revue 40-tal et des publications bien assises comme Ord och bild ou Bonniers litterära magasin (B.L.M.). Cette dernière, fondée en 1932, est devenue un véritable monument national, le symbole d'un certain establishment littéraire. Elle a réussi à survivre jusqu'à nos jours à la foule des jeunes revues éphémères, sa fonction n'étant plus de permettre le débat, mais de témoigner.

7 En Suède, on a toujours été un peu en retard sur le continent en ce qui concerne la littérature, et les jeunes revues littéraires ne font pas exception à la règle. De la nouveauté point trop n'en faut! Cela semble presque normal aux Suédois, prudents et circonspects de nature. La révolte contre les faux cols en littérature n'arrive pas toujours à se défaire elle-même d'un je ne sais quoi de guindé. Si celle-ci est inséparable du modernisme, elle n'est pas spécifique des temps modernes. Ses manifestations relèvent presque toujours des mêmes attitudes : l'antagonisme qui consiste à s'opposer au goût dominant, l'«agonisme" qui caractérise des tendances morbides et autodestructrices, et l'activisme qui se manifeste par un besoin fébrile de changement ${ }^{2}$.

8 D'un point de vue historique la revue Phosphoros (1810-1813) est la première que l'on puisse classer dans la catégorie avant-gardiste. Son héraut était le poète romantique Atterbom qui y appelait à la polémique contre l'académisme. Une aurore nouvelle devait se lever sur une nouvelle époque. Les étudiants d'Uppsala en formaient les militants enthousiastes. Entre 1809 et 1821 douze publications furent ainsi lancées par de jeunes uppsaliens décidés à risquer l'aventure économique d'une jeune revue pour diffuser leurs idées.

Cette floraison de jeunes revues au temps du romantisme n'a cependant été qu'un feu de Bengale, suivi en Suède d'une longue nuit. Il faudra attendre plus d'un siècle avant que les revues de la jeune avant-garde littéraire n'y deviennent une nouvelle fois, à partir de 1930, un "forum pour débutants et débatteurs». Contrairement à ce qui 
s'était passé à l'étranger, en France, en Allemagne, en Angleterre, où les -ismes successifs avaient disposé de leurs propres organes de presse, les écrivains suédois avaient eu tendance, avant cette date, à utiliser plutôt des revues étrangères. Strindberg a publié des articles dans une vingtaine de revues étrangères, françaises, allemandes ou autrichiennes, de même que Selma Lagerlöf, par exemple. Même Pär Lagerkvist, qui était un des premiers grands écrivains suédois à saisir l'importance de la révolution moderniste littéraire ${ }^{3}$, n'a eu que des contacts éphémères avec des revues Scandinaves comme flamman (1917-1921) en Suède ou Klingen (1917-1920) au Danemark, les premières qui cherchaient à diffuser en Scandinavie le message de l'art nouveau.

En Suède les jeunes écrivains se tournaient volontiers alors vers des organes de presse socialistes ou anarchistes ${ }^{4}$, malgré le peu de compréhension que ces derniers montraient en général pour les manifestes littéraires proprement dits. La revue anarchiste Brand était la seule à ouvrir largement ses colonnes aux expérimentations littéraires des jeunes, de ceux qui se considéraient comme des prolétaires en littérature.

11 Après 1918 la Suède continua donc à rester un peu en retrait du modernisme international en littérature. Les jeunes auteurs, comme ceux du groupe De gröna (Les Verts) formés autour de Eyvind Johnson, cherchaient avant tout à donner libre cours à leur agressivité sociale. Ils se réunissaient d'ailleurs dans la Maison du Peuple de Klara à Stockholm. Leur revue Vår Nutid, publiée à partir de 1920, n'a connu que six numéros. On n'y rencontre pas encore cette aspiration à des formes et à une langue nouvelles, qui est une des caractéristiques essentielles du modernisme.

12 En effet, la littérature suédoise, et nordique en général, de même que la critique littéraire de ces pays, ont montré pendant très longtemps un réel scepticisme face aux tendances de renouveau stylistique propres au modernisme et face à la chronologie littéraire, aux -ismes, en usage dans d'autres pays. En Scandinavie on classe la littérature par décennies, plus que par rapport aux -ismes dominants 5 . Edit Södergran, influencée par Nietzsche et par le modernisme russe, a été une des premières au $\mathrm{XX}^{\mathrm{e}}$ siècle à ouvrir la voie de la révolution formelle en Suède. Elle a été la figure de proue, sans l'avoir cherché sans doute, du modernisme finlandais suédophone. Celui-ci a été le premier en Scandinavie, à travers des revues comme Ultra (1922, 7 numéros) ou Quosego (1928, 4 numéros) et sous l'impulsion d'écrivains comme Rabbe Enckell, Elmer Diktonius ou Gunnar Björling, à défendre les droits des jeunes et à inaugurer la voie du renouveau littéraire ${ }^{6}$.

13 Les années 1930 marquent la percée décisive du modernisme en Suède. Cette décennie voit surgir un nouveau porte-bannière, Artur Lundkvist, qui essaiera enfin de créer autour de lui un véritable front moderniste pour une révolution littéraire fondamentale. Peu d'écrivains importants s'engagent cependant réellement à ses côtés, en dehors du groupe des Fem unga (Les cinq jeunes) qu'il dirige et dont font partie Erik Asklund, Josef Kjellgren, Harry Martinson et Gustav Sandgren. Pour faire connaître son groupe, Lundkvist se hasarde à contacter la grande maison d'édition Bonnier. Celle-ci se montre assez réticente pour financer une revue d'avant-garde, mais accepte de publier une sorte d'anthologie de textes programmes Fem unga. Celle-ci paraît fin 1929, tentative purement provisoire aux yeux de Lundkvist. Parcourant alors l'Europe, celui- 
ci s'intéresse énormément aux expériences littéraires en France, aux publications de la revue Transitions dont il écrit qu'elle "plante des signaux et montre des directions à suivre $»^{7}$. Il prend nettement conscience qu'une action concertée par delà les frontières est nécessaire, en littérature comme en politique. «On ne peut pas faire la révolution mondiale tout seul, écrit-il, mais on peut y participer dans son coin. Un coin nordique » 8 .

Le groupe des cinq jeunes s'étoffe assez rapidement, car le prestige de Lundkvist est grand déjà. De nouveaux visages arrivent, des jeunes talents qui brûlent de se faire un nom en littérature. Stellan Arvidson, Karin Boye, Johannes Edfelt, Ivar Lo-Johansson en deviendront les plus célèbres. La direction de la revue Brand leur laisse encore pendant quelques mois la disponibilité de sa page littéraire. Mais les frictions ne tardent pas à se manifester. Ces jeunes révolutionnaires ne peuvent supporter de dépendre du bon vouloir ou du goût littéraire d'un simple journaliste. Encore moins de la prudente réserve d'un éditeur. Bonniers ne consent toujours qu'à publier une anthologie, quelque chose qui se vend bien, en somme. Le recueil Modem lyrik qui paraît en 1931, ouvre, certes, une large fenêtre sur le lyrisme de ces jeunes modernistes, mais laisse aussi une place à des représentants d'un lyrisme plus traditionnel comme Hjalmar Gullberg. Cela ne saurait satisfaire personne, car les jeunes ont envie d'avoir leur manifeste à eux, de renverser quelques vieux meubles.

Et de fait, la Suède connaît alors une véritable et subite inflation de jeunes revues littéraires, qui se succèdent, se soutiennent ou se combattent au rythme des écoles, des chapelles ou des coteries littéraires. Parmi la quinzaine de ces revues ${ }^{9}$, certaines n'ont pas dépassé le stade confidentiel, d'autres ont eu, ou ont encore, un destin plus enviable. Spektrum (1931-1935) a été une des revues les plus célèbres. Elle a lancé le jeune poète Gunnar Ekelöf et fait connaître Harry Martinson au grand public. Fönstret, créé en 1930 à l'occasion de l'exposition de Stockholm, est sans doute la jeune revue littéraire qui a connu la longévité la plus exceptionnelle. Elle est entrée dans le cercle des grandes et continue de nos jours encore à alimenter le débat littéraire en Suède. Les étudiants, comme toujours, et les maisons d'édition sont parmi les éléments moteurs. Fronten (1931, 34 numéros), publié par Bonniers avec Sven Stolpe comme rédacteur en chef, traite de littérature et de politique, cherchant la polémique à tout prix. Ateneum et Presens sont les éphémères revues animées par des groupes d'étudiants. Il y a même des revues qui s'affichent ouvertement réactionnaires, Janus (1934-1937), une revue mensuelle idéologique, et Klingan (1934-1935, 9 numéros), dont l'objectif était de " combattre le primitivisme».

Artur Lundkvist se devait d'être présent dans ce florilège de jeunes revues. Il avait songé à un titre un peu exotique, Karavan; cette revue, dont il réussit à publier cinq numéros (1934-1935), bénéficie du soutien de Bonniers. Les « cinq jeunes » en sont les rédacteurs principaux. Son ambition était de prendre la relève de Spektrum et de faire mieux connaître la littérature étrangère contemporaine, française et anglaise surtout. Les jeunes modernistes autour de Lundkvist avaient alors une conscience aiguë, comme jadis les romantiques, d'être appelés à transformer le monde. Lundkvist était resté en contact et en communion d'idées avec la fédération anarcho-syndicaliste de Stockholm, dont le grand pape Albert Jensen plaidait avec sa fougue habituelle la cause de l'anarchisme, seul garant de la victoire du modernisme.

Cette victoire éclate effectivement en Suède durant les années 1940-1950. Quinze nouvelles revues d'avant-garde en soulignent la portée et l'ampleur ${ }^{10}$, malgré 
l'isolement culturel dans lequel la Suède se trouvait encore au début, à cause de la Seconde Guerre mondiale, et malgré le fait que le concept même de modernisme continue à être l'objet d'âpres discussions. La transition avec l'époque précédente se fait sans heurts, souvent avec les mêmes acteurs. Artur Lundkvist réussit, une fois de plus, à entraîner Bonniers dans l'aventure d'un nouvel organe littéraire Horisont (1941, 5 numéros), qui prend modèle sur Karavan, avec les mêmes collaborateurs et les mêmes objectifs d'ouverture à l'étranger. De nouveaux noms y apparaissent aussi, Erik Lindegren, Johannes Edfelt, Karl Vennberg. On s'intéresse à Kafka, enfin, on traduit Faulkner, Garcia Lorca, Rilke.

Mais le véritable âge d'or du modernisme ne dure que quelques années, de 1944 à 1947, autour de la revue 40-tal. Avec elle, le modernisme suédois prend enfin ses distances avec la politique, avec le marxisme. La disparition simultanée de Kulturfront (1941-1944, 22 numéros) en cette année 1944, revue liée au mouvement communiste international où l'on trouvait les signatures de Martin Andersen Nexö, Jan Fridegård ou Moa Martinson, en est du reste la confirmation. Avec le soutien actif de Bonniers, 40-tal se veut avant tout un organe de débats esthétiques et littéraires, regroupant les principaux 40-talistes autour de Lennart Göthberg, Stig Dagerman, Werner Aspenström et Karl Vennberg, sans programme littéraire précis, sans manifeste à défendre. Ce qui prédomine dans la revue, c'est un pessimisme généralisé. La psychanalyse semble déjà avoir fait son temps, l'humanisme n'est plus qu'un « oiseau sans nid » (W. Aspenström), l'anarchisme, tout comme l'esthétisme, une simple mode plus qu'un point de vue à défendre. La polémique néanmoins va bon train dans la revue, essentiellement pour ou contre la poésie moderniste. Vennberg et Lindegren en étaient devenus les grandsprêtres. Stig Dagerman se penchait avec délectation sur les insolubles conflits entre éthique et esthétique. 40-tal était vraiment le forum ouvert à toutes les contributions, sans épuiser aucun des sujets.

Le champ des débats restait, en effet, largement ouvert aux autres revues concurrentes, Stil/Presens (1944-1947) qui marquait l'arrivée de Göteborg dans le débat littéraire, et Sesam (1946-1947) qui se voulait une réaction contre le trop-plein de nihilisme en littérature. Mais la disparition simultanée en 1947 de ces trois revues, 40-tal, Presens et Sesam, signifie d'une certaine manière qu'après la fin du tout-politique le temps des polémiques est, lui aussi, révolu.

Prisma (1948-1950), édité par Norstedt avec Erik Lindegren comme rédacteur principal, cherche alors à présenter une synthèse culturelle du modernisme international, en littérature, musique, beaux-arts, danse et film. Une synthèse déjà, comme si on s'apprêtait à poser les premières pierres du musée du modernisme !

21 Cette revue d'une très haute tenue, une des plus intéressantes du point de vue de la qualité de ses publications, offre une large place à la réflexion sur la mission de l'art dans la restauration sociale, après les désastres de la guerre. La promotion de talents nouveaux reste davantage l'apanage de la revue Utsikt (1948-1950, 29 numéros), dont Bonniers confie la responsabilité à Axel Liffner, avec la mission expresse de prendre la suite de 40-tal. Mais le modernisme à tous crins de la revue ne fait déjà plus recette, alors que le succès de sa concurrente directe, All världens berättare ne cesse de se confirmer. Cette dernière, lancée déjà en 1945 par les éditions Åhlén et Åkerlund pour rehausser le niveau et la réputation des lectures populaires tant critiquées par les modernistes, s'était spécialisée dans la publication de nouvelles de très grande qualité, suédoises ou étrangères. Son rédacteur K.J. Rådström, sans dédaigner les 
expérimentations formelles, se laisse surtout guider dans ses choix par l'intérêt narratif des textes. Très rapidement la revue va atteindre le tirage respectable de 40000 exemplaires. Un record absolu pour la Suède. Au bout de dix-huit années d'existence elle sera reprise par B.L.M.

L'habitude de publier des revues littéraires étant ainsi devenue une des composantes du paysage littéraire suédois, on continue donc allègrement après 1950 à en créer de nouvelles, bien que l'avant-gardisme eût déjà beaucoup de plomb dans l'aile. Durant cette décennie, dix-sept nouvelles revues littéraires tentent leur chance, rien de moins, tant la frénésie du nouveau semble s'être ancrée dans les esprits ${ }^{11}$.

Tidsbilden, ambitionnant d'être la revue culturelle du mouvement ouvrier, veut mobiliser des noms célèbres, mais cesse subitement de paraître au bout de 4 numéros (20.1.51-10.2.51). Bonniers, toujours à l'affût des nouveautés et se souvenant de l'impact de la défunte revue 40-tal, favorise une publication nouvelle, 50-tal, dont seul le nom sera, en fait, prestigieux. Cette revue éphémère (10 numéros), « dont le baptême fut pure folie» selon Lasse Bergström, donne, en effet, l'impression d'une simple anthologie, un type de publication que cette maison d'édition semble affectionner particulièrement. Elle reste terne face à d'autres revues comme Metamorfos qui, sans dépasser le stade de la confidentialité, surent davantage galvaniser de jeunes talents.

La recherche fébrile de nouvelles orientations à laquelle on assiste alors ne fait que confirmer l'essoufflement du modernisme suédois. Certains tentent de renouer avec le provincialisme (Provins, Synpunkt, Ultima, une revue laponne), se souvenant peut-être que Selma Lagerlöf, Fröding, Heidenstam y avaient jadis puisé la sève de leur génie universel. On cherche surtout à se rendre indépendant des éditeurs. Des groupes de jeunes écrivains se réunissent dans des cafés, créent leur propre système d'édition. Paravan (1953-1957), une revue d'art et de politique, en est un des exemples.

Schismen (1954), qui veut distribuer ses critiques à droite comme à gauche, se singularise en ne publiant que sous des pseudonymes, mais ne dépasse pas les trois premiers numéros. Svenska ungförfattartidningen excelle dans la pure prétention en n'acceptant que des contributions d'écrivains en dessous de seize ans! Plus sérieuse était, en revanche, la revue Kulturkontakt (1954-1958) qui groupait autour de Ture Nerman un certain nombre d'écrivains engagés dans la lutte contre le totalitarisme communiste.

26 Certes, les talents littéraires sont alors trop nombreux dans le pays pour que ne se révèlent pas, çà et là, quelques excellentes publications d'avant-garde. Salamander, imprimé à Malmö en 1955, en est un exemple, mais ne réussit pas à sortir plus de trois numéros. Les noms très connus des 40-talistes, Karl Vennberg ou Stig Carlson entre autres, pouvaient maintenant se satisfaire de leur propre notoriété, sans s'engager davantage à côté de nouveaux débatteurs et débutants.

Tribun (1955) en fait l'amère expérience, ne trouvant nulle part assez d'oxygène pour aller au-delà du premier numéro. Le seul programme qui s'avère encore payant pour l'avant-garde des modernistes - ou ne devrait-on pas plutôt parler de leur arrièregarde? - c'est l'intérêt commercial, le soutien de l'éditeur. Bonniers encourage le critique littéraire Göran Palm dans sa tentative, la dernière du genre, de rivaliser avec la renommée et l'impact littéraire des 40-talistes en finançant la publication de Upptakt 
(1955-1958, 20 numéros). Mais c'est un travail d'épigone, tout simplement, témoin mélancolique d'une époque qui s'achève. Le modernisme, c'est sûr maintenant, a vécu. Bientôt surgira le débat, nouveau et attendu, autour d'un éventuel postmodernisme.

Toute cette longue série de revues d'avant-garde littéraire aura néanmoins contribué avec panache, malgré leur déconfiture économique quasi générale et leur longévité maigrichonne, à doter la Suède et la Scandinavie d'une foule de jeunes talents littéraires. À cet égard le modernisme y représente une période à nulle autre pareille. Il a profité du renouveau d'intérêt pour la littérature qui s'est manifesté dans le grand public après 1945. Son engagement antitotalitaire contre les extrémismes de droite comme de gauche, est brandi comme un drapeau et se manifeste déjà dans la valeur symbolique, ou provocante, des titres que se donnent toutes ces jeunes revues. Le seul élément faisant un peu contrepoids à l'exaltation périodique qui se manifestait avec allégresse dans la nef aventureuse du modernisme, ce fut le rôle de mécène intéressé que jouaient les maisons d'édition, et tout spécialement Bonniers.

Si les vagues de la nouvelle esthétique, du nouvel art de vie du modernisme ont déferlé sur la Suède, au gré des lunes, avec un peu de retard sur le continent, leur effet n'en fut pas moins révolutionnaire. Les revues d'avant-garde ont été dans le vaste flottement, les fluctuations et les essais de recomposition qui s'en suivirent, les messagères de nouvelles théories poétologiques, englobant les multiples aspects de la vie, et le forum de leurs exercices pratiques. Elles nous apparaissent comme les charnières d'une époque en mutation. Leur rôle n'est d'ailleurs jamais terminé, il durera tant que durera la remise en question de l'héritage. Même si le vocabulaire change, même si dans les années 1970 on parlera plutôt de "revues alternatives", la jeune revue littéraire d'avant-garde, comme le phénix, renaîtra constamment encore de ses cendres.

\section{NOTES}

1. A. Lundkvist : Lettre à Erik Asklund du 23.8.1928: "Nous sommes quelques-uns qui depuis longtemps poursuivent le projet de créer une revue de jeune littérature ».

2. Cf. Renato Poggioli, Teoria dell'arte avanguardia, 1962.

3. Cf. notamment l'essai de P. Lagerkvist, Ordkonst och bildkonst.

4. Des revues comme Brand, Fram, Frihet ou Stormklockan ont joué un rôle important dans l'évolution intellectuelle et culturelle de la Suède durant les quatre premières décennies du $\mathrm{XX}^{\mathrm{e}}$ siècle.

5. Cf. à ce sujet Fritz Paul, Stil und Epochenwandel im Spiegel skandinavischer Literaturzeitschriften, Antrittsvorlesung an der Universität Bochum, 1973.

6. Durant la période 1920-1930 sept revue littéraires d'avant-garde ont été publiées en Suède, avec plus ou moins de bonheur, dont, outre celles déjà mentionnées, les revues Orfeus et Juvenes dum sumus publiées par les étudiants de Stockholm réunis autour de Sven Stolpe, Svart pä vitt et Tidens magasin lancé par les Ed. Tiden.

7. Lettre à Stina Aronson du 23.6.1930.

8. Ibid. 
9. Il s'agit des revues suivantes : Fönstret, Kontakt, Spektrum, Votum, Fronten,Ateneum, Presens, Janus, Klingan, Mänsklighet, Kulturfront, Karavan, Nordeuropa,U.P.A.

10. Il s'agit des revues suivantes : Horisont, Realisten, Kulturfront, Création, Ars, 40-tal, Stil, Presens, All världens berättare, Sesam, 1945, Prisma, Poesi, Utsikt, Medan lagrarna gro, Quo vadis.

11. Il s'agit des revues suivantes : Tidsbilden, 50-tal, Arena, Metamorfos, Odyssé, Provins, Synpunkt, Paravan, Schismen, Upsalagazetten, Svenska ungförfattartidnin-gen, Kulturkontakt, Salamander, Tribun, Upptakt, Hjo L.M., Siesta, Ultima.

\section{RÉSUMÉS}

"Un forum pour débuts et débats ", c'est ainsi que l'écrivain Axel Liffner a défini en 1948 la jeune revue littéraire d'avant-garde. Celle-ci constitue l'arme par excellence des jeunes générations littéraires, de celles qui cherchent leur inspiration dans un contexte international, en Scandinavie surtout. Le destin de la jeune revue est étroitement fonction de son message ; peu savent se renouveler assez pour durer. En Suède, il faut attendre les années 1930 pour que se renouvelle la floraison des jeunes revues que le pays avait connu un siècle plus tôt, au temps du romantisme. Les décennies 1920-1930 et 1930-1940 voient s'opérer la percée du modernisme, avec chaque fois une quinzaine de jeunes revues, sous la conduite d'écrivains de premier plan comme Arthur Lundkvist ou Eric Lindegren. Les étudiants et les maisons d'édition en sont souvent les éléments moteurs.

La victoire du modernisme en Suède éclate enfin, à travers des revues comme 40-tal, durant les années 1940-1950. Son âge d'or est cependant de courte durée. Les nouvelles revues d'avantgarde continuent encore après cette date à se multiplier, dix-sept entre 1950 et 1960. Mais, malgré leur recherche d'une nouvelle justification, elles ne font que confirmer l'essoufflement du modernisme. À l'actif de toutes ces revues d'avant-garde littéraire reste cependant un fait essentiel: elles ont contribué, malgré leur déconfiture économique et leur longévité maigrichonne, à doter la Suède d'une foule de jeunes talents littéraires. Elles sont comme les charnières d'une époque en mutation, et leur rôle en tant que messagères de nouvelles théories poétologiques ne sera jamais complètement achevé.

"Ein Forum für Debüt und Debatte ", so hat Axel Liffner 1948 die junge avantgardistische Zeitschrift definiert. Diese bildet die bevorzugteste Waffe in der Hand der jungen Schriftsteller, derjenigen die, besonders in Skandinavien, ihre Muster im Ausland suchen. Das Schicksal der jungen avantgardistischen Zeitschrift steht in engem Zusammenhang mit der neuen Botschaft, die sie verbreiten will ; nur wenige vermögen sich so zu erneuern, dass sie auf die Dauer bestehen können. In Schweden hat sich die Blüte der jungen literarischen Zeitschriften, die das Land zur Zeit der Romantik gekannt hatte, erst wieder gegen das Jahr 1930 erneuert. Der Durchbruch des Modernismus geschieht dort in den Jahrzehnten 1920-1930 und 1930-1940, unter dem Impuls von jedesmal mehr als 15 neuen Zeitschriften und dem Ansporn von Schriftstellern ersten Ranges wie Artur Lundkvist oder Eric Lindegren. Die Studenten und die wichtigsten Verlage sind oft diejenigen, welche dem Ganzen den entscheidenden Schwung geben.

Schweden erlebt aber den endgültigen Sieg des Modernismus erst in den Jahren 1940-50, dank dem Einfluss von gewissen jungen Zeitschriften, wie 40-tal z.B. Die Blütezeit ist allerdings von kurzer Dauer, obwohl das Feld der laufenden esthetischen Debatte weit offen bleibt. Die jungen avantgardistischen Zeitschriften schiessen auch nach dieser Periode weiter aus dem Boden, wie 
Pilze, mehr als 17 zwischen 1950 und 1960. Aber durch ihr hektisches Suchen nach einer neuen Berechtigung bestätigen sie nur die Dekadenz des Modernismus. Eines muss jedoch dieser langen Reihe von jungen modernistischen Zeitschriften, die sich über Jahrzehnte hinstreckt, zu gut gerechnet werden; sie haben, trotz ihrem finanziellen Fiasko und ihrer gewöhnlichen Kurzlebigkeit, entschieden dazu beigetragen, im Land eine Menge von jungen literarischen Talenten zu fördern. Sie stehen sozusagen jedesmal am Wendepunkt einer neuen Periode, und ihre Rolle als Verkünderinnen einer neuen Poetologie wird immer noch aktuell bleiden.

\section{AUTEUR}

\section{GEORGES UEBERSCHLAG}

Université Charles-de-Gaulle - Lille III 